\title{
Equivariant quantization of spin systems
}

\author{
J.-Ph. Michel ${ }^{1}$ \\ Institut Camille Jordan, Université Claude Bernard Lyon 1, 43 boulevard du 11 novembre 1918, \\ F-69622 Villeurbanne Cedex France \\ and \\ University of Luxembourg, Campus Kirchberg, Mathematics Research Unit, 6, rue Richard \\ Coudenhove-Kalergi, L-1359 Luxembourg City, Grand Duchy of Luxembourg.
}

\begin{abstract}
We investigate the geometric and conformally equivariant quantizations of the supercotangent bundle of a pseudo-Riemannian manifold $(M, g)$, which is a model for the phase space of a classical spin particle. This is a short review of our previous works [1, 2].
\end{abstract}

Keywords: conformal geometry, pseudomechanics, spin geometry, equivariant quantization. MSC:53A30,53C27,53D50,53Z05,58A50,70H50.

\section{INTRODUCTION}

Quantization is born with quantum mechanics, as the fundamental attempt to establish a correspondence between the mathematical structures of classical and quantum mechanics, presented in the following table.

\begin{tabular}{c|c|c} 
& classical & quantum \\
\hline Phase space & symplectic manifold $(\mathscr{M}, \omega)$ & Hilbert space $\mathscr{H}$ \\
\hline Observables & Poisson algebra $A \subset \mathscr{C}^{\infty}(\mathscr{M})$ & associative algebra $\mathscr{A} \subset \mathscr{L}(\mathscr{H})$ \\
\hline Symmetries & Lie subalgebra $\mathfrak{g} \subset \operatorname{ham}(\mathscr{M}, \omega)$ & Lie subalgebra $\mathfrak{g} \subset \mathrm{u}(\mathscr{H})$.
\end{tabular}

One of the most celebrated quantization procedure is the geometric quantization $[3,4]$, whose main draw-back is its too small set of quantizable observables. Equivariant quantization $[5,6]$ aims to overcome this issue for systems admitting a configuration space $M$ with a large enough group $G$ of (local) symmetries, as the projective or conformal group. More precisely, the inverse of the obtained quantization map is a $G$-equivariant symbol map on $M$, from differential operators to symmetric tensors.

We present here the equivariant quantization of spin systems whose configuration space is a spin manifold $M$ endowed with a metric $g$ of signature $(p, q)$. This suppose to introduce a framework for classical mechanics of spin systems, namely the supercotangent bundle of $(M, g)$ [7, 8] endowed with its canonical symplectic structure [9]. We recover some of the main objects of spin geometry via its geometric quantization, and the privileged status of conformal transformations of $(M, g)$ is highlighted. Restricting us

${ }^{1}$ I thank the Luxembourgian NRF for support via the AFR grant PDR-09-063. 
to a conformally flat manifold, we describe explicitly the action of $o(p+1, q+1)$ on the space of classical and quantum observables, plus on a related space of tensors. We state then our main results on the existence and uniqueness of conformally equivariant quantization and superization, which are isomorphisms between these three $\mathrm{o}(p+1, q+1)$ modules. Some applications are given. We refer to $[1,2]$ for more details and proofs.

\section{FROM CLASSICAL TO QUANTUM SPIN SYSTEMS}

The quantum framework for spin systems with configuration space $(M, g)$ is wellknown: the state space $\mathscr{H}$ is obtained by completion of the space of sections of the spinor bundle $\mathrm{S} \rightarrow M$, and we choose the algebra $\mathscr{D}(M, \mathrm{~S})$ of spinor differential operators as space of quantum observables. Its usual algebra of symbols is $\operatorname{Pol}\left(T^{*} M\right) \otimes$ $\Gamma(\mathbb{C l}(M, g))$, the tensor product of the space of functions on $T^{*} M$, which are polynomial in the fiber variables, with the space of sections of the complex Clifford bundle of $(M, g)$. Replacing $\Gamma(\mathbb{C l}(M, g))$ by its graded counterpart, namely the algebra of complex differential forms $\Omega_{\mathbb{C}}(M)$, we end up with a superalgebra of functions on the supercotangent bundle of $M$. That provides us with the algebra of symbols for $\mathscr{D}(M, \mathrm{~S})$ w.r.t. its bifiltration [8], as well as with the classical setting for a spin system on $M$.

\section{Supercotangent bundle and pseudomechanics}

The supercotangent bundle of the manifold $M$ is $\mathscr{M}=T^{*} M \oplus \Pi T M$, i.e. the direct sum of the cotangent bundle and the tangent bundle with reverse parity. Thus, its superalgebra of functions is $\mathscr{C}^{\infty}\left(T^{*} M\right) \otimes \Omega(M)$, generated locally by coordinates $\left(x^{i}, p_{i}, \xi^{i}\right)$, where $\xi^{i}$ identifies with $d x^{i}$. The general study of symplectic supermanifolds by Rothstein [9] proves that a symplectic structure on $\mathscr{M}$ is equivalent to the data of a metric and a compatible connexion on $M$. As a consequence, to any pseudo-Riemannian manifold $(M, g)$ corresponds a canonical symplectic form $\omega$ on $\mathscr{M}$, given by

$$
\omega=d \alpha \quad \text { and } \quad \alpha=p_{i} d x^{i}+\frac{\hbar}{2 \mathrm{i}} g_{i j} \xi^{i} d^{\nabla} \xi^{j}
$$

where $d^{\nabla}$ is the covariant differential w.r.t. the Levi-Civita connexion of $g$. The spin components are defined as $S^{i j}=\frac{\hbar}{i} \xi^{i} \xi^{j}$ so that, together with the Poisson bracket associated to $\omega$, they generate a Lie algebra isomorphic to $\mathrm{o}(p, q)$. The equations of motion of a spin particle in an exterior electromagnetic field can be easily recovered in that framework $[1,7]$, and also the coupling of the spin with the gravitation $[1,10]$. Thus, the Hamiltonian flows of the kinetic energy $g^{i j} p_{i} p_{j}$ leads to the Papapetrou's equations [11],

$$
\begin{aligned}
& \dot{x}^{j} \nabla_{j} \dot{x}^{i}=-\frac{1}{2} g^{i k} R(S)_{j k} \dot{x}^{j} \\
& \dot{x}^{k} \nabla_{k} S^{i j}=0
\end{aligned}
$$


where the spin happens to be coupled with the curvature via $R(S)_{j k}=g_{i m} R_{l j k}^{i} S^{j k}$, with $\left(R_{l j k}^{i}\right)$ the components of the Riemann tensor.

\section{Spin geometry via geometric quantization}

Thanks to geometric quantization, we can built the main objects of spin geometry from $(\mathscr{M}, \omega)$ endowed with a polarization, i.e. a Lagrangian distribution. Upon topological restrictions on $M$, the vertical polarization of the cotangent bundle of $M$ can be completed by a maximal isotropic complex distribution for $g$ on $\Pi T M$, to give a polarization on $(\mathscr{M}, \omega)$. In the simplest case of a Riemannian metric, the geometric quantization leads then to the construction of Hitchin [12] for the spinor bundle $S$ of a pseudo-Hermitian manifold $(M, g)$, where spinor fields identify with antiholomorphic differential forms tensorized with square root of the volume form of $(M, g)$.

Besides, for Darboux coordinates $\left(x^{i}, \tilde{p}_{i}, \tilde{\xi}^{i}\right)$ of $(\mathscr{M}, \omega)$, the quantum map ${ }^{2}$ satisfies

$$
\mathscr{Q}\left(x^{i}\right)=x^{i}, \quad \mathscr{Q}\left(\tilde{p}_{i}\right)=\frac{\hbar}{\mathrm{i}} \partial_{i} \quad \text { and } \quad \mathscr{Q}\left(\tilde{\xi}^{i}\right)=\frac{\tilde{\gamma}^{i}}{\sqrt{2}},
$$

where $\tilde{\gamma}^{i}$ is a Clifford matrix for the flat metric given by $\left(\eta_{i j}\right)=\mathbb{I}_{p} \oplus-\mathbb{I}_{q}$. Let us remind that the vector fields on $M$ can be lifted to Hamiltonian vector fields on $T^{*} M$, giving rise to a tautological momentum map $J$, such that $J_{X}=p_{i} X^{i}$ for $X=X^{i} \partial_{i} \in \operatorname{Vect}(M)$. This map can be lifted to $\mathscr{M}$ and quantized,

$$
\mathscr{Q}\left(J_{X}\right)=\frac{\hbar}{\mathrm{i}} \nabla_{X}
$$

giving rise to an essential object of the spin geometry: the covariant derivative of spinors.

\section{Conformal geometry of the supercotangent and spinor bundles}

The symplectic structure of the supercotangent bundle $\mathscr{M}$ depends on a metric $g$ on $M$, as a consequence the natural lift to $\mathscr{M}$ of $X \in \operatorname{Vect}(M)$ does not preserve the potential 1-form $\alpha$. Moreover, the condition $L_{\tilde{X}} \alpha=0$ does not uniquely determine the lift $\tilde{X}$ of $X$. One way out is to impose the further condition $L_{\tilde{X}} \beta \propto \beta$, where $d \beta=g_{i j} d^{\nabla} \xi^{i} \wedge d x^{j}$ is the odd symplectic form on $\Pi T M$. The lift is then unique but exists only if $X$ is a conformal Killing vector field. We refer to it later as $\tilde{X}$. Let us notice that, as expected, the momentum of an infinitesimal rotation $X_{i j}$ is,

$$
\mathscr{J}_{X_{i j}}=p_{i} x_{j}-x_{j} p_{i}+S_{i j}
$$

Remarkably, the quantization of the momentum map $\mathscr{J}$ leads to

$$
\mathscr{Q}\left(\mathscr{J}_{X}\right)=\frac{\hbar}{\mathrm{i}} \mathrm{L}_{X}
$$

\footnotetext{
${ }^{2}$ We consider here that $\mathscr{Q}$ takes its values in usual spinor fields rather than spinor half-densities.
} 
the Lie derivative of spinors introduced by Kosmann [13], well-defined precisely for conformal Killing vector fields. Via geometric quantization, we get thus the conformal geometry of the spinor bundle of $(M, g)$ out of the one of its supercotangent bundle.

\section{CONFORMALLY EQUIVARIANT QUANTIZATION}

We suppose from now on that $(M, g)$ is a conformally flat spin manifold, i.e. $g_{i j}=F \eta_{i j}$ for some positive function $F$. The conformal Killing vector fields on $(M, g)$ generate then a Lie algebra isomorphic to $\mathrm{o}(p+1, q+1)$, which is the one of infinitesimal conformal transformations of $\left(\mathbb{R}^{n}, \eta\right)$. The aim is to compare the action of those vector fields on $\mathrm{D}(M, \mathrm{~S})$, the algebra of spinor differential operators, with those on its algebras of symbols, i.e. to compare their o $(p+1, q+1)$-module structures.

\section{Conformal geometry of spinor differential operators and of their symbols}

Let us define the space of $\lambda$-densities by $\mathscr{F}^{\lambda}=\Gamma\left(\left|\Lambda^{n} T^{*} M\right|^{\otimes \lambda}\right)$, with $\lambda \in \mathbb{R}$ and $n=\operatorname{dim} M$. Instead of $\mathrm{D}(M, \mathrm{~S})$, we will rather study the two parameters family of $\mathrm{o}(p+1, q+1)$-modules $\left(\mathrm{D}^{\lambda, \mu}\right)$. Each of those modules is defined as the space of differential operators $D: \Gamma(\mathrm{S}) \otimes \mathscr{F}^{\lambda} \rightarrow \Gamma(\mathrm{S}) \otimes \mathscr{F}^{\mu}$, endowed with the adjoint action

$$
\mathscr{L}_{X}^{\lambda, \mu} D=\mathrm{L}_{X}^{\lambda} D-\mathrm{L}_{X}^{\mu} D
$$

where $\mathrm{L}_{X}^{\lambda}=\mathrm{L}_{X}+\lambda \operatorname{Div}(X)$ is the action of $X$ on $\Gamma(\mathrm{S}) \otimes \mathscr{F}$. The corresponding $\mathrm{o}(p+1, q+1)$-module of classical observables is naturally the space $\mathscr{S}^{\delta}[\xi]=\operatorname{Pol}\left(T^{*} M\right) \otimes \Omega_{\mathbb{C}}(M) \otimes \mathscr{F}^{\delta}$, with $\delta=\mu-\lambda$, endowed with the Hamiltonian action

$$
L_{X}^{\delta}=\tilde{X}+\delta \operatorname{Div}(X) .
$$

The explicit expressions of these both classical and quantum actions have been computed in [2], showing that $D^{\lambda, \mu}$ and $\mathscr{S}^{\delta}[\xi]$ are filtered modules, by the order of differential operators and the degree in the $p$ variables respectively. Their common associated graded module is the module of tensorial symbols $\mathscr{T}^{\delta}[\xi]=\bigoplus_{\kappa=0}^{n} \operatorname{Pol}\left(T^{*} M\right) \otimes \Omega_{\mathbb{C}}^{\kappa} \otimes \mathscr{F}^{\delta-\frac{\kappa}{n}}$, endowed with the natural action on weighted tensors. It identifies to $\operatorname{Pol}\left(T^{*} M\right) \otimes \Omega_{\mathbb{C}}(M)$ as an algebra and to the usual space of symbols $\operatorname{Pol}\left(T^{*} M\right) \otimes \Gamma(\mathbb{C l}(M, g))$ as a module.

\section{Main results}

Let us begin with few definitions. A map is called conformally equivariant if it is an isomorphism of $\mathrm{o}(p+1, q+1)$-modules. Besides, as $\operatorname{Pol}\left(T^{*} M\right)$ is a submodule of 
$\mathscr{T}^{0}[\xi]$, a linear isomorphism $\mathscr{T}^{\delta}[\xi] \rightarrow \mathscr{S}^{\delta}[\xi]$ which preserves the principal symbol ${ }^{3}$ is named a superization, whereas a linear isomorphism $\mathscr{S}^{\delta}[\xi] \rightarrow \mathrm{D}^{\lambda, \mu}$ preserving the principal symbol is a quantization, since it relates classical and quantum observables.

Theorem 1 There exists $I_{\mathrm{S}}^{e} \subset I_{\mathrm{S}} \subset \mathbb{Q}_{+}^{*}$ such that the conformally equivariant superization $\mathrm{S}_{\mathscr{T}}^{\delta}: \mathscr{T}^{\delta}[\xi] \rightarrow \mathscr{S}^{\delta}[\xi]$, exists if $\delta \notin I_{\mathrm{S}}^{e}$ and is unique if $\delta \notin I_{\mathrm{S}}$.

Theorem 2 Let $\delta=\mu-\lambda \in \mathbb{R}$. There exists $I_{\mathscr{Q}}^{e} \subset I_{\mathscr{Q}} \subset \mathbb{Q}_{+}^{*}$ such that the conformally equivariant quantization $\mathscr{Q}^{\lambda, \mu}: \mathscr{S}^{\delta}[\xi] \rightarrow \mathrm{D}^{\lambda, \mu}$, exists and is unique if $\delta \notin I_{\mathscr{Q}}$, and exists for at least one value of $\lambda \in \mathbb{R}$ if $\delta \notin I_{\mathscr{Q}}^{e}$.

The values of $\delta$ for which existence or uniqueness of $\mathscr{Q}^{\lambda, \mu}$ is lost are called resonances. The fact that $\delta=0$ is not a resonance is crucial, thus the conformally equivariant quantization $\mathscr{Q}^{\frac{1}{2}, \frac{1}{2}}$ extends uniquely the quantization map provided by geometric quantization. Let us remark that $\mathscr{Q}^{\lambda, \mu} \circ S_{\mathscr{T}}^{\delta}$ is a particular case of AHS-equivariant quantization [14], but each single map deserves interest, at least from a physical point of view.

The idea of the proofs is the same than in the spinless case [6], and relies on the use of the Casimir operators of each module. To be concrete, we name $C_{\mathscr{T}}$ and $C_{\mathscr{S}}$ those of $\mathscr{T}^{\delta}[\xi]$ and $\mathscr{S}^{\delta}[\xi]$. If the conformally equivariant superization exists, then $C_{\mathscr{S}} \mathrm{S}_{\mathscr{T}}^{\delta}=\mathrm{S}_{\mathscr{T}}^{\delta} C_{\mathscr{T}}$, and in particular every eigenvectors of $C_{\mathscr{T}}$ is send to an eigenvector of $C_{\mathscr{S}}$ with the same eigenvalue and the same principal symbol. The main point is to prove that the eigenvectors of $C_{\mathscr{T}}$ are uniquely determined by their eigenvalue and their principal symbol. Then, if this is the case for those of $C_{\mathscr{S}}$ too, we get the uniqueness of the superization. As $C_{\mathscr{S}}$ is equal to $C_{\mathscr{T}}$ plus an operator lowering the degree in the $p$ variable, this is simply checked by the resolution of triangular systems, and the resonant values of $\delta$ are precisely those leading to degenerated systems. If unique, the map constructed in this way is easily proved to be conformally equivariant.

\section{Some applications}

We give now two applications that we hope to investigate further in forthcoming papers. The first one relies on the explicit formulas for the conformally equivariant superization that we determine in [1] for symbols of degree 1 in $p$. Let us recall that a (conformal) Killing-Yano tensor on $M$ is a skew-symmetric tensor describing higher symmetries of $(M, g)$. As Killing tensors, it generates constant of motion but for spin particles [15]. In fact, a correspondence has been obtained in [16] between Killing-Yano tensors and classical supercharges, which happens to be generalized by the conformally equivariant superization.

Theorem 3 Let $f$ be a skew-symmetric tensor and $P_{f}=f_{j_{1} \ldots j_{\kappa-1}}^{i} \xi^{j_{1}} \ldots \xi^{j_{\kappa-1}} p_{i}$ the associated tensor symbol. Denoting by $\Delta=p_{i} \xi^{i}$, we have

$$
\left\{\Delta, S_{\mathscr{T}}^{0}\left(P_{f}\right)\right\}=0(\propto \Delta) \Longleftrightarrow f \text { is a (conformal) Killing-Yano tensor. }
$$

\footnotetext{
3 That is the higher order term of an element w.r.t. a filtration, here it identifies to a tensor over $M$.
} 
The second application deals with the conformal invariants of the $\mathrm{o}(p+1, q+1)$ modules that we have introduced. Let $R=g^{i j} p_{i} p_{j}$. The Weyl theory of invariants together with the explicit actions of $\mathrm{o}(p+1, q+1)$ on each module leads to the following Theorem.

Theorem 4 The conformal invariants of each family of modules are

1. $\Delta^{a} R^{s} \in \mathscr{T}^{\frac{2 s+a}{n}}[\xi], \quad$ where $s \in \mathbb{N}$ and $a=0,1$.

2. $\Delta R^{s} \in \mathscr{S}^{\frac{2 s+a}{n}}[\xi]$, where $s \in \mathbb{N}$.

3. $\mathscr{Q}^{\lambda, \mu}\left(\Delta R^{s}\right) \in \mathrm{D}^{\frac{n-2 s-1}{2 n}, \frac{n+2 s+1}{2 n}}, \quad$ where $s \in \mathbb{N}$.

In particular, we get the Dirac operator as the lower order conformal invariant of $\left(D^{\lambda, \mu}\right)$. Since the maps $\mathrm{S}_{\mathscr{T}}^{\delta}$ and $\mathscr{Q}^{\lambda, \mu}$ preserve conformal invariance, we deduce from the last Theorem that $\mathrm{S}_{\mathscr{T}}^{\frac{2 s}{n}}$ does not exist and that the module $\mathrm{D}^{\frac{n-2 s-1}{2 n}}, \frac{n+2 s+1}{2 n}$ is exceptional: this the only one in the family $\left(\mathrm{D}^{\lambda, \lambda+\frac{2 s+1}{n}}\right)$ to be isomorphic to its space of symbols $\mathscr{S}^{\frac{2 s+1}{n}}[\xi]$. As a consequence, $\delta=\frac{2 s+1}{n}$ are resonances. In fact, every resonances correspond to some conformal invariants, that will be the matter of our next paper.

\section{ACKNOWLEDGMENTS}

It is a pleasure to acknowledge Christian Duval for his essential guidance, and invaluable discussions. Special thanks are due to Valentin Ovsienko for his constant interest in this work.

\section{REFERENCES}

1. J.-P. Michel, Quantification conformément équivariante des fibrés supercotangents, Ph.D. thesis, Université Aix-Marseille II (2009), electronically available as tel-00425576.

2. J.-P. Michel, arXiv:1004.1595 [math.DG] (2010), URL http://arxiv.org/abs/1004. 1595.

3. B. Kostant, "Quantization and unitary representations. I. Prequantization," in Lectures in modern analysis and applications, III, Springer, Berlin, 1970, pp. 87-208. Lecture Notes in Math., Vol. 170.

4. J.-M. Souriau, Structure des systèmes dynamiques, Maîtrise de mathématiques, Dunod, Paris, 1970 (C) 1969).

5. P. B. A. Lecomte, and V. Y. Ovsienko, Lett. Math. Phys. 49, 173-196 (1999), ISSN 0377-9017.

6. C. Duval, P. B. A. Lecomte, and V. Y. Ovsienko, Ann. Inst. Fourier (Grenoble) 49, 1999-2029 (1999), ISSN 0373-0956.

7. F. A. Berezin, and M. S. Marinov, Annals of Physics 104, 336-362 (1977).

8. E. Getzler, Comm. Math. Phys. 92, 163-178 (1983), ISSN 0010-3616.

9. M. Rothstein, "The structure of supersymplectic supermanifolds," in Differential geometric methods in theoretical physics (Rapallo, 1990), Springer, Berlin, 1991, vol. 375 of Lecture Notes in Phys., pp. 331-343.

10. F. Ravndal, Phys. Rev. D (3) 21, 2823-2832 (1980), ISSN 0556-2821.

11. A. Papapetrou, Proc. Roy. Soc. London. Ser. A. 209, 248-258 (1951), ISSN 0962-8444.

12. N. Hitchin, Advances in Math. 14, 1-55 (1974), ISSN 0001-8708.

13. Y. Kosmann, Ann. Mat. Pura Appl. (4) 91, 317-395 (1972), ISSN 0003-4622. 
14. A. Cap, and J. Silhan, Advances in Mathematics 224, 1717 - 1734 (2010), ISSN 0001-8708, URL http://wWw.sciencedirect.com/science/article/B6W9F-4YB84S8-2/ 2/fd5e6f2a132680c5188f94fdc702f2c6.

15. G. W. Gibbons, R. H. Rietdijk, and J. W. van Holten, Nuclear Phys. B 404, 42-64 (1993), ISSN 0550-3213.

16. M. Tanimoto, Nuclear Phys. B 442, 549-560 (1995), ISSN 0550-3213. 